\title{
Observation of Anisotropy in the Arrival Direction Distribution of TeV Cosmic Rays with HAWC
}

\author{
Segev Y. BenZvi, ${ }^{a}$ Daniel W. Fiorino ${ }^{* b}$ and Stefan Westerhoff ${ }^{b}$ for the HAWC \\ Collaboration ${ }^{c}$ \\ ${ }^{a}$ Department of Physics and Astronomy, University of Rochester, Rochester, NY, USA \\ ${ }^{b}$ Department of Physics and WIPAC, University of Wisconsin-Madison, Madison, WI, USA \\ ${ }^{c}$ For a complete author list, see www.hawc-observatory.org/collaboration/icrc2015.php. \\ E-mail: fiorino@wipac.wisc.edu
}

The High-Altitude Water Cherenkov (HAWC) Observatory, located $4100 \mathrm{~m}$ above sea level near Sierra Negra $\left(19^{\circ} \mathrm{N}\right)$ in Mexico, is sensitive to gamma rays and cosmic rays at $\mathrm{TeV}$ energies. The arrival direction distribution of cosmic rays at these energies shows significant anisotropy on several angular scales, with a relative intensity ranging between $10^{-3}$ and $10^{-4}$. We present the results of a study of cosmic-ray anisotropy based on more than 86 billion cosmic-ray air showers recorded with HAWC since June 2013. The HAWC cosmic-ray sky map, which has a median energy of $2 \mathrm{TeV}$, exhibits several regions of significantly enhanced cosmic-ray flux. We present the energy dependence of the anisotropy and the cosmic-ray spectrum in the regions of significant excess.

The 34th International Cosmic Ray Conference,

30 July- 6 August, 2015

The Hague, The Netherlands

\footnotetext{
${ }^{*}$ Speaker.
} 


\section{Introduction}

The HAWC Observatory has recently completed construction at $4100 \mathrm{~m}$ above sea level near Puebla, Mexico. The observatory, located at $19^{\circ} \mathrm{N}$ latitude, is designed to study the sky in gamma rays and cosmic rays between $50 \mathrm{GeV}$ and $100 \mathrm{TeV}$. While cosmic rays are the major source of background in the gamma-ray analysis, the distribution of the arrival directions of the cosmic rays is itself of significant interest. Cosmic rays with rigidities of several TV strongly scatter on Galactic magnetic fields $\left(r_{g} \approx 0.001 \mathrm{pc}\right)$, scrambling their initial direction many times before reaching Earth. Despite this, the last decade of experiments has reported stable $10^{-3}-10^{-4}$ sidereal anisotropy in the arrival direction distribution of $\mathrm{TeV}$ cosmic rays in both hemispheres (see Ref [1] and references therein). The anisotropy has been observed at large $\left(>60^{\circ}\right)$ and small angular scales by multiple experiments.

At $\mathrm{TeV}$ energies, the large-scale structure is dominated by dipole and quadrupole moments of consistent phase throughout all experiments. These studies require year-long observations to cancel the effects of the Solar dipole. This study will not be presented with this half-year data set. After removal of the large-scale structure, significant small-scale structure appears, most notably a region of excess near $\left(\alpha=60^{\circ}, \delta=-5^{\circ}\right)$ [2,3]. Small-scale deficits do not appear to be significant features and may be an artifact of the removal of the large-scale structure.

The origin of the anisotropy is not well understood. It has been suggested that weak dipole or dipole-like features should be a consequence of the diffusion of cosmic rays from nearby sources in the galaxy $[4,5]$. This hypothesis is currently of considerable interest $[6,7,8]$. Excesses may be the result of magnetically connected cosmic-ray accelerators or something more exotic [9, 10]. Smallscale anisotropy may also arise as a feature of turbulent scattering of large-scale anisotropy $[11,12]$.

The newly finshed HAWC detector observes a larger portion of the sky with a higher recorded event rate than any high-energy cosmic-ray detector before it. This proceeding serves as an update to Ref. [1]. These data cover a region of the sky previously unobserved by experiments operating in the northern and southern hemispheres. In these proceedings we present measurements of anisotropy on small angular scales with an emphasis on the energy-dependence of the signficant features. We compare the observed anisotropy with previous measurements of the northern and southern skies.

\section{Data Set}

The HAWC Observatory is a $22,000 \mathrm{~m}^{2}$ array of closepacked water Cherenkov detectors (WCDs). Each WCD consists of a cylindrical steel water tank $4.5 \mathrm{~m}$ in height and $7.3 \mathrm{~m}$ in diameter. The complete detector now comprises 300 WCDs with 1200 PMTs.

The analysis presented in this paper uses the data collected during the operation of 111 WCDs (HAWC-111) between June 16, 2013 and July 8, 2014. Triggers for gamma-ray and cosmicray air showers were formed with a simple multiplicity trigger which requires $>15$ PMTs to be above threshold within a sliding time window of $150 \mathrm{~ns}$. The trigger rate in HAWC-111 was approximately $15 \mathrm{kHz}$. The data were reconstructed offline, and with $111 \mathrm{WCDs}$ the angular resolution of the air shower reconstruction was approximately $1.0^{\circ}$. This is sufficient to observe the $5^{\circ}-180^{\circ}$ features in the anisotropy of the cosmic rays. A cut in the fractional number of 
PMTs $>6 \%$ is used to remove poorly reconstructed events from the data. During this period the detector collected $8.6 \times 10^{10}$ well-reconstructed events. Only continuous sidereal days of data were chosen for these analyses in order to reduce the bias of uneven exposure along right ascension. The resultant data set has a livetime of 181 days. Using the detector simulation we estimate that the median energy of the data set is about $2 \mathrm{TeV}$.

\section{Analysis}

To search for anisotropy, we directly compute the relative intensity as a function of equatorial coordinates $(\alpha, \delta)$. We begin by binning the sky into an equal-area grid with a resolution of $0.2^{\circ}$ per bin using the HEALPix library. A binned data map $N(\alpha, \delta)_{i}$ is used to store the arrival directions of air showers recorded by the detector for each angular bin $i$.

To produce residual maps of the anisotropy of the arrival directions of the cosmic rays, we must have a description of the arrival direction distribution if the data arrived isotropically at Earth, $\langle N\rangle(\alpha, \delta)_{i}$. We calculate this expected flux from the data themselves in order to account for rate variations in both time and viewing angle of the data. The spatial distribution of events is unique to a detector configuration and stable for periods exceeding 24 hours. The all-sky rate varies in Solar time by $\sim 5 \%$ and approximates a sinusoid.

The reference map $\langle N\rangle(\alpha, \delta)_{i}$ is produced using the direct integration technique described in Ref. [13], adapted for the HEALPix grid. In brief, we proceed by collecting all events recorded during a predefined time period $\Delta t$ and integrate the local arrival direction distribution against the detector event rate. The method effectively smooths out the true arrival direction distribution in right ascension on angular scales of roughly $\Delta t \cdot 15^{\circ} \mathrm{hr}^{-1}$ such that the analysis is only sensitive to structures smaller than this characteristic angular scale. The direct integration procedure also compensates for variations in the detector rate while preserving the event distribution in declination. Once the reference map is obtained, we calculate the deviations from isotropy by computing the relative intensity

$$
\delta I(\alpha, \delta)_{i}=\frac{\Delta N}{\langle N\rangle}=\frac{N(\alpha, \delta)_{i}-\langle N\rangle(\alpha, \delta)_{i}}{\langle N\rangle(\alpha, \delta)_{i}}
$$

which gives the amplitude of deviations from the isotropic expectation in each angular bin $i$. The significance of the deviation can be calculated using the method of Li and Ma [14].

This analysis method can be sensitive to any maximum angular scale through the choice of $\Delta t$. Due to the sampling of the reference map along lines of right ascension, the maximum angular scales as $(\cos \delta)^{-1}$. Only a choice of 24 hours ensures a uniform angular scale as a function of declination. To remove larger structure, a multipole fit can be subtracted to access lower angular scales while preserving the maximum angular scale throughout the map.

\section{Measurement of the Small-scale Anisotropy}

The analysis was carried out on HAWC-111 data using $\Delta t=24 \mathrm{hr}$ to obtain sensitivity to all angular features equally over the sky. The results are plotted in Figure 1 in relative intensity. The data have been smoothed using a $10^{\circ}$ top-hat function to make the clustering of arrival directions 


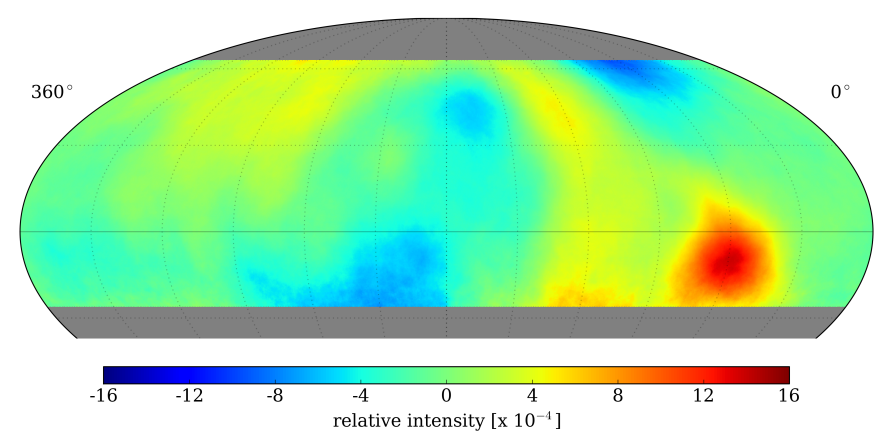

Figure 1: Relative intensity of the cosmic-ray flux for 181 days of HAWC-111, in equatorial coordinates. A period $\Delta t=24 \mathrm{hr}$ is used to access the largest features present in the map. The map is shown with $10^{\circ}$ smoothing applied.

readily apparent. At this time we do not provide an in-depth description of the observed large-scale features. The measurement in Fig. 1 is a combination of sidereal anisotropy and the Solar dipole effect which causes an excess of cosmic rays in the direction of the Earth's motion around the Sun. The two are difficult to disentangle without a full year of data in which the signals make a complete transit in the other reference frame. The Solar contamination in this data set has been confirmed by observing significant signal in a sky map made in an unphysical coordinate system ("anti-sidereal" frame).

The large-scale signals can be subtracted from the map in Fig. 1 using a multipole fit to all multipole moments $\ell \leq \ell_{\max }$. Any small-scale residual from the Solar signal should be negligible $\left(<10^{-5}\right)$, so we are left with the small-scale structure in the sidereal frame. The residual structure will contain power at angular scales less than the $180^{\circ} / \ell_{\max }$. To reveal angular scale $<60^{\circ}$, a choice of $\ell_{\max }=3$ was made. The fit of all moments with $\ell \leq 3$ in the map in Fig. 1 were was subtracted to produce the relative intensity and significance maps shown in Figure 2. Again, a $10^{\circ}$ smoothing has been applied, so only pre-trials significances are shown. The estimated number of trials is at most equal to the number of independent bins $\left(\sim 10^{5}\right)$, but is in fact much smaller, since we are not performing a blind search; all excess regions have been perviously observed by other experiments. The subtraction of the fit removes angular power in $\ell \leq 3$ without influencing the higher order multipoles as evidenced by a comparison of the angular power spectra in Figure 3.

With the trials factor taken into account, three signficant features remain, the strong region of excess flux at $\left(\alpha=60^{\circ}, \delta=-10^{\circ}\right)$, the band of excess along $\alpha=120^{\circ}$, and a weaker excess at $\left(\alpha=240^{\circ}, \delta=45^{\circ}\right)$. These excesses lie coincident with the $10^{\circ}-20^{\circ}$ regions of cosmic-ray access first observed by Milagro (Regions A and B in [2]) and a third region observed only by ARGO-YBJ [3] (Region C). The strongest excess, with a pre-trial significance of $20.6 \sigma$, is found at $\alpha=58.4^{\circ}$ and $\delta=-8.8^{\circ}$ and corresponds to Region A in the Milagro map. The relative intensity of the excess in this region peaks at $(7.9 \pm 0.4 \pm 0.4) \times 10^{-4}$, where the first error is statistical and the second error is systematic. The median cosmic-ray energy at this declination is $2.1 \mathrm{TeV}$. For comparison, we fit a 2-D Gaussian function to the relative intensity map around Region A. The center is located at $\alpha=59.2^{\circ} \pm 0.6^{\circ}$ and $\delta=-7.2^{\circ} \pm 0.7^{\circ}$, with an amplitude of $(8.7 \pm 0.9) \times 10^{-4}$. 

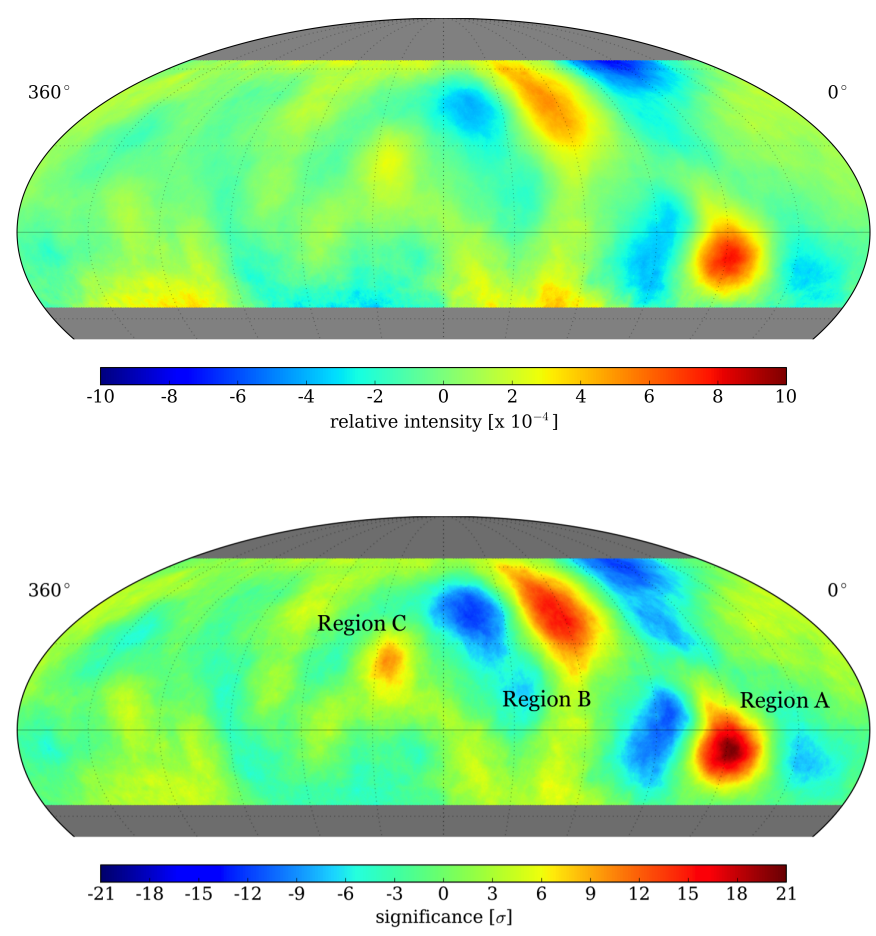

Figure 2: Relative intensity (top) and pre-trial significance (bottom) of the cosmic-ray flux after fit and subtraction of the dipole, quadrupole, and octupole terms from the map shown in Fig. 1. The map is shown with $10^{\circ}$ smoothing applied.

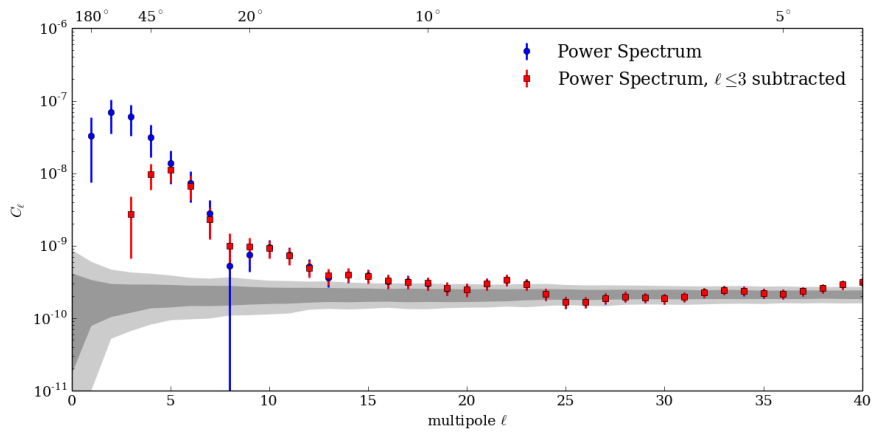

Figure 3: Angular power spectra of the unsmoothed relative intensity map before (blue) and after (red) subtracting the large-scale structure $(\ell \leq 3)$. Gray errors bands show the $68 \%$ and $95 \%$ spread of the $C_{\ell}$ for isotropic data sets. Comparing the band to the data shows which ell-modes significantly contribute to the sky map. The error bars on the $C_{\ell}$ are the square root of the variances returned by a fit using a power spectrum estimator (PolSpice). 
The width is nearly symmetric in $\alpha$ and $\delta$, approximately $7.6^{\circ} \pm 1.2^{\circ}$.

The elongated excess around $\alpha=120^{\circ}$, coincident with Region $\mathrm{B}$, extends over a wide range of declinations. It is most significant at $\left(119.5^{\circ}, 38.9^{\circ}\right)$ with a pre-trial significance of $15.6 \sigma$ and a relative intensity of $(5.3 \pm 0.4 \pm 0.2) \times 10^{-4}$. A third excess region, Region $\mathrm{C}$, is centered at $\alpha=206.0^{\circ}$ and $\delta=23.8^{\circ}$ with a pre-trial significance of $10.5 \sigma$ and a peak relative intensity of $(2.8 \pm 0.3 \pm 0.7) \times 10^{-4}$. The median cosmic-ray energy at this declination is $2.0 \mathrm{TeV}$.

There are also regions of strong deficits visible, typically on both sides of the strong excess regions. The appearance of these deficit regions, correlated with the excess regions, is likely an artifact caused by imperfections in the multipole fit.

\section{Study of Region A}

An early version of energy estimation has been developed for studying the HAWC data set as a function of energy. Seven energy bins are separated by cutting on both the incident zenith angle and the fraction of the detector that observed a cosmic-ray air shower. The energies of these bins are determined from simulation. At this time, the energy bins are heavily overlapping, still, a clear evolution of Region A as a function of energy is identifiable. This region exhibits a harder spectrum than background.

We estimate the statistical significance of the hardness the in Region A spectrum by comparing the slope of a linear fit of $\delta I$ versus $\log (E)$ in Figure 4 to similar fits performed at many random locations in the field of view. The random locations excluded a $15^{\circ}$ circle centered on Regions $\mathrm{A}, \mathrm{B}$, and $\mathrm{C}$. The distribution of slopes for the random locations follows a Gaussian distribution centered at zero with a width of $0.9 \times 10^{-4}$. The best fit slope at the position of Region A is $(3.8 \pm$ $1.1) \times 10^{-4}, 4.2 \sigma$ away from the mean. The reduced $\chi^{2}$ of a fit to a sloped-line is 1.16 compared to 5.66 for a horizontal line. This further supports a spectrum that is harder than background.

A zoomed view of Region A in four energy-proxy bins (the final four bins of Fig. 4 were combined) is provided in Figure 5. In addition to the spectral steepening, this figure shows the excess extending to higher declination as a function of energy, consistent with the ARGO observation.

\section{Discussion}

This half-year HAWC-111 data set is already one third as large as the Milagro 8-year data set and one fourth of the ARGO-YBJ 5-year data set. We have found significant small-scale structure that coincides with 3 of the 4 previously published regions of cosmic-ray excess. In general, there is excellent agreement between the observed excesses and deficits of previous measurements by ARGO-YBJ and Milagro in the Northern Hemisphere.

Region $\mathrm{A}$ at $\alpha=60^{\circ}$ appears to have two sub-regions in the $\mathrm{TeV}$ range. At higher energies $\left(>10 \mathrm{TeV}\right.$ ), the northern part at $\delta=5^{\circ}$ appears. Below this, the southern part at $\delta=-5^{\circ}$ is dominant. Milagro saw the northern part of Region A, but only observed down to $\delta=-5^{\circ}$. There is a slight indication of the southern part of Region A in the unpublished relative intensity maps. Both the HAWC and ARGO maps see the change with energy in Region A. This suggests that the hardening of the spectrum of Region A is coincident with a northernly movement of the excess or the emergence of an entirely new region of excess. 


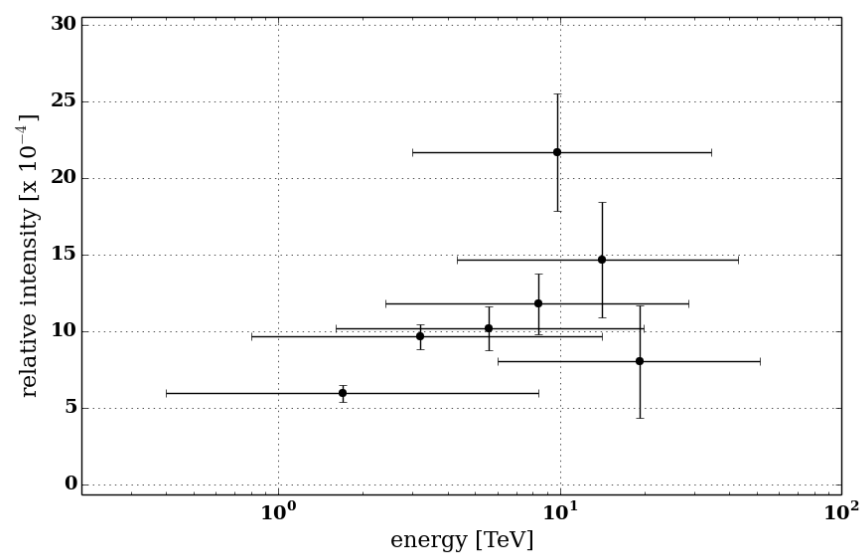

Figure 4: Spectrum of Region A in relative intensity in different energy-proxy bins. The energies of the data were determined from simulation. The error bars on the median energy values correspond to a $68 \%$ containing interval.
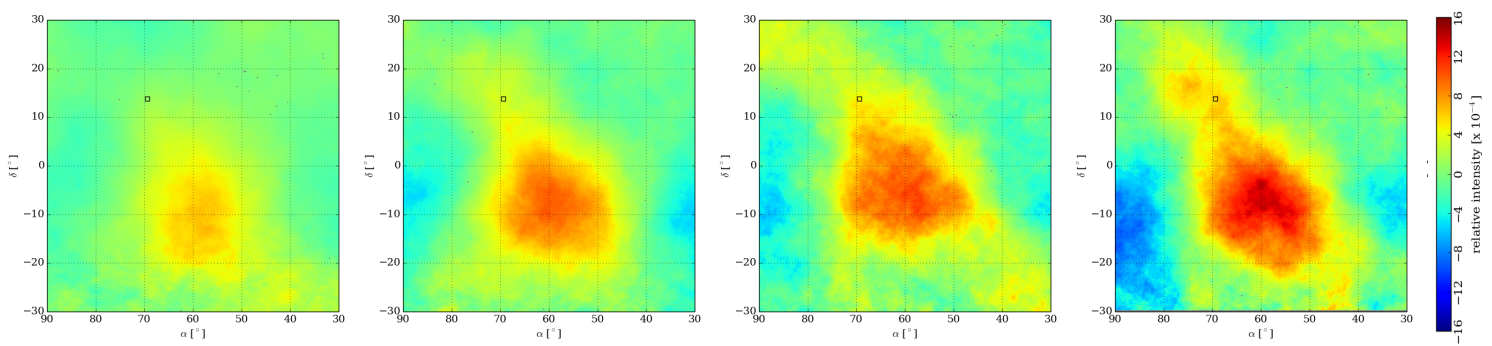

Figure 5: Relative intensity of Region A for 4 different energy-proxy bins. The square mark denotes the location of the centroid of Region A as reported by Milagro $\left(\alpha=69.4^{\circ}, \delta=13.8^{\circ}\right)$ at $10 \mathrm{TeV}$. The median energy of the data in each plot from left to right is $1.7_{-1.3}^{+6.6} \mathrm{TeV}, 3.2_{-2.4}^{+10.9} \mathrm{TeV}, 5.6_{-3.9}^{+14.2} \mathrm{TeV}$, and $14.1_{-9.9}^{+28.7} \mathrm{TeV}$.

Region B at $\alpha=120^{\circ}$ is visible at all declinations observed by HAWC and seems to follow the edge of the large-scale sidereal deficit. The extension of the excess to higher declinations is notable because this is not observed to high significance in the Milagro data, even though Milagro was located at a higher latitude $\left(35^{\circ} \mathrm{N}\right)$. The significance maps published by ARGO also extend to high declinations, and these maps indicate that the Region B excess extends northward.

Region $\mathrm{C}$ at $\alpha=205^{\circ}$ is not significant in the Milagro data, but the ARGO collaboration has observed a hot spot at the same location of similar shape and intensity. ARGO also observed a fourth significant region at $\alpha=280^{\circ}$ which appears as a sub threshold excess in the HAWC map. Continued observation with HAWC will help confirm this as a true excess.

A comparison between data from HAWC-111 and IceCube (20 TeV median) in the Southern Hemisphere is very interesting because connecting the northern and southern measurements would eliminate biases from partial sky coverage. No clear connection of the small-scale anisotropy that is present in both hemispheres has been made yet. The published IceCube maps are of higher energy than HAWC and other northern measurements. Because of this, a first effort at combining HAWC and IceCube data uses cuts to bring their median energies closer to a central value [15]. 
Ten years after the first sky maps that charted the cosmic-ray anisotropy, the state of the art in this field has moved towards studying significant cosmic-ray features as a function of rigidity. Precision measurements of cosmic-ray anisotropy are readily obtained from air shower detectors, such as HAWC. Initial improvements to the resolution and range of measured cosmic-ray energies using these detectors have revealed energy-dependent behavior of the anisotropy. Further studies of this phenomenon may provide insight into local cosmic-ray production and propagation or may even become a tool for studying exotic physics.

\section{References}

[1] HAWC Collaboration, A. Abeysekara et al., Observation of Small-scale Anisotropy in the Arrival Direction Distribution of TeV Cosmic Rays with HAWC, The Astrophysical Journal 796 (2014) 108, [arXiv:1408.4805].

[2] Milagro Collaboration, A. A. Abdo et al., The Large Scale Cosmic-Ray Anisotropy as Observed with Milagro, Astrophys. J. 698 (2009) 2121-2130, [arXiv: 0806.2293 ].

[3] ARGO-YBJ Collaboration, B. Bartoli et al., Medium scale anisotropy in the TeV cosmic ray flux observed by ARGO-YBJ, Phys.Rev. D88 (2013) 082001, [arXiv: 1309 . 6182].

[4] A. D. Erlykin and A. W. Wolfendale, The anisotropy of galactic cosmic rays as a product of stochastic supernova explosions, Astropart. Phys. 25 (2006) 183-194, [astro-ph / 0601290 ].

[5] P. Blasi and E. Amato, Diffusive propagation of cosmic rays from supernova remnants in the Galaxy. II: anisotropy, JCAP 1201 (2012) 011, [arXiv: 1105 . 4529].

[6] M. Pohl and D. Eichler, Understanding TeV-Band Cosmic-Ray Anisotropy, Astrophys. J. 766 (2013) 4, [arXiv:1208.5338].

[7] L. G. Sveshnikova, O. N. Strelnikova, and V. S. Ptuskin, Spectrum and Anisotropy of Cosmic Rays at TeV-PeV-energies and Contribution of Nearby Sources, Astropart. Phys. 50-52 (2013) 33-46, [arXiv:1301.2028].

[8] P. Mertsch and S. Funk, Solution to the Cosmic Ray Anisotropy Problem, Phys. Rev. Lett. 114 (Jan, 2015) 021101.

[9] M. Ángeles Pérez-García, K. Kotera, and J. Silk, Anisotropy in Cosmic rays from internal transitions in neutron stars, Nucl. Instrum. Meth. A742 (2014) 237-240, [arXiv:1309.1852].

[10] J. P. Harding, The TeV Cosmic-Ray Anisotropy from Local Dark Matter Annihilation, arXiv:1307.6537 (2013) [arXiv:1307.6537].

[11] G. Giacinti and G. Sigl, Local Magnetic Turbulence and TeV-PeV Cosmic Ray Anisotropies, Phys. Rev. Lett. 109 (2012) 071101, [arXiv:1111.2536].

[12] M. Ahlers, Anomalous Anisotropies of Cosmic Rays from Turbulent Magnetic Fields, Phys. Rev. Lett. 112 (2014) 021101, [arXiv:1310.5712].

[13] Milagro Collaboration, R. W. Atkins et al., Observation of TeV gamma-rays from the Crab nebula with MILAGRO using a new background rejection technique, Astrophys. J. 595 (2003) 803-811, [astro-ph/0305308].

[14] T.-P. Li and Y.-Q. Ma, Analysis methods for results in gamma-ray astronomy, Astrophys. J. 272 (1983) 317-324.

[15] HAWC Collaboration, J. Dìaz-Velez and D. Fiorino, Full-Sky Analysis of Cosmic-Ray Anisotropy with Ice Cube and HAWC, in Proc. 34th ICRC, (The Hague, The Netherlands), August, 2015. 